\title{
Metas de aprendizaje y rasgos de personalidad como predictores del bienestar en cadetes de una institución policial
}

\author{
Liliana Paola Nieri¹ e Iván Fritzler²
}

\begin{abstract}
RESUMEN
La presente investigación tuvo como objetivo investigar si la motivación académica y los rasgos de personalidad predicen el bienestar de los cadetes de policía, a la vez que se analizaron diferencias en el bienestar según sexo y edad. La muestra estuvo compuesta por 411 cadetes con una media de edad de 24.08 años $(D T=3.18)$ pertenecientes a una institución policial estatal. Los instrumentos utilizados fueron: el BFI (Big Five Inventory), EMA - M (Escala de Metas de Aprendizaje), MHC-SF (Mental Health Continuum - Short Form) y un cuestionario sociodemográfico. Los resultados obtenidos permitieron comprobar que los mayores predictores positivos del bienestar fueron extraversión $(t=$ $3.412 ; \beta=.157 ; p<.01)$; agradabilidad $(t=2.901 ; \beta=.144 ; \mathrm{p}<.01)$; responsabilidad $(t=$ $3.851 ; \beta=.195 ; p<.001$ ) y metas en relación con el aprendizaje y el dominio de los conocimientos $(t=2.446 ; \beta=.103 ; p<.05)$. De igual manera se halló que el único predictor negativo fue el rasgo de personalidad neuroticismo $(t=-3.555 ; \beta=-.184 ; p$ $<.01)$. Asimismo, se encontró que existen diferencias estadísticamente significativas en la dimensión bienestar social $\left(t_{410)}=1.816 ; p<.05\right)$ entre hombres y mujeres. Finalmente, se halló que la dimensión bienestar psicológico variaba según edad $\left(F_{(2,411)} ; p<.05\right)$, donde el grupo de mayor edad presenta mayores niveles de bienestar psicológico. En cuanto al

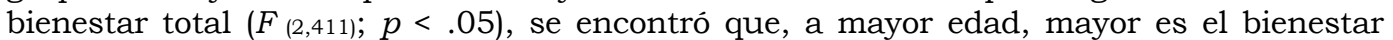
total. En vista de los resultados obtenidos, puede afirmarse que tanto la personalidad como la motivación por el aprendizaje son variables que predicen el bienestar total de los estudiantes. Asimismo, se deja constancia que los niveles de bienestar varian en función del género y la edad.
\end{abstract}

Palabras clave: bienestar, metas de aprendizaje, personalidad, cadetes, policía.

\section{Learning goals and personality traits as predictors of the well-being of cadets in a police institution}

\begin{abstract}
The aim of the present study was to investigate whether academic motivation and personality traits predict the welfare of police cadets, while analyzing differences in wellbeing according to sex and age. The sample consisted of 411 cadets with an average age of 24.08 years $(S D=3.18)$ belonging to a state police institution. The instruments used were BFI (Big Five Inventory), EMA - M (Learning Goal Scale), MHC- SF (Mental Health Continuum - Short Form) and a sociodemographic questionnaire. The results obtained showed that the greatest positive predictors of well-being were extraversion $(t=3.412 ; \beta$ $=.157 ; p<.01)$; pleasantness $(t=2,901 ; \beta=.144 ; \mathrm{p}<.01)$; responsibility $(t=3.851 ; \beta=$ $195 ; p<.01)$ and goals in relation to learning and mastery of knowledge $(t=2.446 ; \beta$ $=.103 ; p<.05)$. Similarly, it was found that the only negative predictor was the personality trait neuroticism $(t=-3.555 ; \beta=-.184 ; p<.01)$. Likewise, it was found that there are statistically significant differences in the social well-being $\left(t_{(410)}\right)=1.816, p$
\end{abstract}

\footnotetext{
Consejo Nacional de Investigaciones Cientificas y Técnicas (CONICET), Argentina; liliananieri@hotmail.com; https://orcid.org/0000-0002-3615-7515

2 Universidad Argentina de la Empresa, Argentina; ivanfritzler@gmail.com; https://orcid.org/0000-0002$1978-4357$
} 
$<.05)$ between men and women. Finally, it was found that the psychological well-being varied according to age $\left(F_{(2,411)}, p<.05\right)$ where the older age group presented higher levels of psychological well-being. In terms of total well-being $\left(F_{(2,411)}, p<.05\right)$, it was found that the greater the age, the greater the total well-being. In view of the results obtained, it can be affirmed that both the personality and the motivation for learning are variables that predict the total well-being of the students. Likewise, it is recorded that levels of wellbeing vary according to gender and age.

Keywords: learning goals, well-being, personality, police cadets.

En general, la revisión de la bibliografia permite observar un desarrollo muy escaso de investigaciones en cadetes de policía. Especificamente en el ámbito policial, la literatura muestra una tendencia a investigar aspectos patológicos de la personalidad (Wang et al., 2010), coincidiendo con un modelo de ser humano carente de aspectos positivos, resultante del foco exclusivo en la patología que dominó mucho tiempo en la psicología (Seligman \& Csikszentmihalyi, 2000). Sin embargo, las nuevas lineas de investigación han considerado los avances teóricos de la psicología y la evolución de la función institucional de las fuerzas armadas, hecho por el cual han incorporado nuevas variables (Adler, 2013; Bardera, García-Silgo \& Pastor, 2014; Benatuil $\&$ Castro Solano, 2007; Castro Solano, 2005). En este sentido, se evidencia la necesidad de reforzar estas líneas de investigación estudiando con mayor profundidad aspectos psicológicos más bien positivos, considerando en este caso aquellos factores asociados con el bienestar de los aspirantes a las fuerzas armadas.

El bienestar se configura como un indicador relevante de la calidad de vida, no solo a nivel individual, sino también a nivel colectivo (Siegrist, 2003). Arraigadas en la tradición filosófica, dos han sido las corrientes predominantes para su estudio psicológico: una relacionada con el bienestar subjetivo (hedónico) y otra con el bienestar psicológico (eudamónico), donde el punto en el que ambas convergen da cuenta del funcionamiento psicológico positivo (Keyes, Shmotkin \& Ryff, 2002; Ryan \& Deci, 2001).

En este contexto, el bienestar se define como una amplia aproximación que incluye el bienestar emocional, entendido en términos de afecto positivo y satisfacción con la vida (Diener, 1984), el bienestar social, entendido como "la valoración que hacemos de las circunstancias y el funcionamiento dentro de la sociedad" (Keyes, 1998, p. 122), y el bienestar psicológico, entendido en términos de autoaceptación, relaciones positivas, autonomía, dominio del entorno, propósito en la vida y crecimiento personal (Ryff, 1989). Es en este sentido donde cobra relevancia la identificación de los factores que predicen el bienestar para alcanzar una comprensión más profunda del mismo y de los factores que pueden afectar su consecución (Grant, Langan-Fox \& Anglim, 2009).

Diversos estudios han demostrado que los estudiantes presentan vulnerabilidad a bajos niveles de bienestar, ya sea porque el inicio de una carrera genera estrés y ansiedad, ya sea por las presiones a las que el estudiante está expuesto para obtener cierto grado académico y luego encontrar un trabajo acorde con su formación que le permita sustento económico (Burris, Brechting, Salsman \& Carlson, 2009; Cooke, Bewick, Barkham, Bradley \& Audin, 2006). 
Por otra parte, los rasgos de personalidad son otro factor que influye sobre el bienestar (Schmutte \& Ryff, 1997). En consonancia con ello, el modelo de los cinco grandes explica la personalidad en términos de cinco rasgos, a saber: extraversión, agradabilidad, responsabilidad, neuroticismo y apertura a la experiencia (Costa \& McCrae, 1992; John, Donahue \& Kentle, 1991). Aunque estos cinco rasgos no cubren la totalidad de la variabilidad de la personalidad, la enmarcan de manera general, hecho por el que resultan relevantes para describir sus relaciones con el bienestar (Compton, 1998).

En este marco, Schmutte y Ryff (1997) han propuesto cierto nivel de correspondencia entre las dimensiones del bienestar y los rasgos de personalidad, donde las personas con una alta autoaceptación deberían verse influenciadas por las tendencias emocionales básicas de rasgos como el neuroticismo y la extroversión. Además, han mencionado que aquellos individuos con alta responsabilidad presentarian mayor autonomía y dominio del entorno, lo que daría lugar una mejor autoaceptación. Por otra parte, los altos niveles de agradabilidad se relacionan con las relaciones positivas, donde los individuos establecen vínculos satisfactorios basados en el apoyo y la calidez. A su vez, las autoras mencionan que la extraversión es otro facilitador de las relaciones positivas.

De igual manera, Schmutte y Ryff (1997) mencionan que los individuos con una alta puntuación en responsabilidad experimentan mayor dominio del entorno y crecimiento personal, a la vez que un bajo neuroticismo, alta extraversión y apertura a la experiencia repercuten en una mayor autonomía. A su vez, las autoras advierten que los individuos con alta extraversión presentan mayor crecimiento personal, a la vez que altos niveles de conciencia se relacionan con la capacidad para establecer un propósito en la vida.

No obstante, vale hacer la salvedad de que son relativamente escasos los estudios teóricos que se han llevado a cabo sobre la relación entre el bienestar y los rasgos de personalidad, aunque puede destacarse que en estos se ha observado que la responsabilidad, el neuroticismo y la extraversión son los que más predicen las dimensiones del bienestar (Bardi \& Ryff, 2007; Grant et al., 2009; Keyes et al., 2002; Schmutte \& Ryff, 1997).

Del mismo modo, considerando que la población en estudio se configura por cadetes de policia, es decir, estudiantes, otro factor relevante son las metas de aprendizaje, entendidas como una actitud caracterizada por el involucramiento permanente y a largo plazo, donde se genera compromiso y se sostiene la actitud en el tiempo (Ames, 1992). En este contexto, mientras que un alumno intrinsecamente motivado asume la responsabilidad de una tarea o bien por su propio interés, o bien por la satisfacción que le genera el aprendizaje que resulta congruente con sus propias expectativas, lo opuesto sucede con un alumno extrínsecamente motivado, donde la responsabilidad se asume sobre la base de la obtención de algún reconocimiento o la evitación de algún castigo o consecuencia negativa, externa a la actividad en sí (Lepper, 1988). Sobre esto, Castro Solano (2005) aclara que es en el primer caso donde se da una mayor calidad de las experiencias de aprendizaje, teniendo en consideración que los estudiantes están más orientados con móviles en relación con el dominio y adquisición del conocimiento que por metas externas a ellos, de manera tal que se adquiere sentido de dominio y competencia.

A la vez, Kasser y Ryan (1996) mencionan que hay asociación entre la motivación intrínseca y el bienestar, teniendo en cuenta que este último se compone por el funcionamiento autónomo del individuo, coincidiendo de esta 
manera con la teoría de la autodeterminación, donde se identifican diferentes tipos de motivación con distintas consecuencias para el aprendizaje, el rendimiento y el bienestar psicológico (Ryan \& Deci, 2000). En este sentido, la relevancia de las metas de aprendizaje respecto al bienestar tiene base en la teoría de la autodeterminación, en la cual se afirma que la satisfacción de las necesidades psicológicas básicas como la autonomía, la competencia y el compromiso en las relaciones promueven el bienestar (Deci \& Ryan, 2000).

Finalmente, en lo que concierne a bienestar específicamente, vale destacar que diversas han sido las investigaciones que han indagado esta variable en función del sexo y la edad. Respecto a ello, la literatura tiende a manifestar un aumento del bienestar conforme avanza la vida, a la vez que se destacan diferencias en el mismo en función del sexo, donde las mujeres poseen mayores niveles de bienestar que los hombres (García Alandete, 2013; Mayordomo, Sales, Satorres \& Meléndez, 2016; Muratori, Zubieta, Bobowik, Ubillos \& González, 2015; Zubieta, Muratori \& Fernández, 2012).

¿Existen predictores del bienestar psicológico en aspirantes a cadetes de policía? ¿Cuáles son las características de personalidad de un aspirante que deben considerarse para que ejerza la profesión con un bienestar adecuado? Estas interrogantes fueron la base de la presente investigación, la cual se configuró en torno al objetivo principal de investigar si las variables metas de aprendizaje (metas en relación con el aprendizaje y dominio de los conocimientos; metas aversivas-externas y metas de lucimiento) y personalidad (extraversión, agradabilidad, responsabilidad, neuroticismo y apertura a la experiencia) predicen el bienestar de los cadetes de policías. Asimismo, como objetivos específicos, se analizó si existían diferencias significativas en el bienestar de los cadetes considerando el sexo y la edad.

La hipótesis planteada para esta investigación fue: las variables rasgos de personalidad (extraversión y responsabilidad) y metas de aprendizaje (dominio de conocimiento) predicen de manera positiva el bienestar de los cadetes aspirantes a policias

En este sentido, conocer los factores que influyen en el bienestar de los cadetes de policía resulta relevante al configurarse como un aporte de valor a considerar para el desarrollo de intervenciones y programas conforme a las necesidades específicas de la población policial.

\section{MÉTODO}

\section{Tipo y diseño de estudio}

Diseño no experimental, de corte transversal; tipo de estudio correlacional.

\section{Participantes}

Se recolectó una muestra no probabilística de sujetos voluntarios en la que participaron 411 cadetes de policía, de los cuales el 59\% $(n=243)$ era de sexo masculino y el $41 \%$ de sexo femenino $(n=169)$, con un promedio de edad de 24.08 años $(D E=3.18)$.

En cuanto al lugar de residencia, todos los participantes eran argentinos nativos residentes de la Provincia de Buenos Aires, el 79.4\% $(n=327)$ del Gran Buenos Aires, el 14.3\% $(n=59)$ de la Ciudad Autónoma de Buenos Aires y el $6.3 \%(n=26)$ del interior de Buenos Aires. 
En relación con el estado civil, el 61.4\% $(n=253)$ se encontraba soltero, el $19.2 \%(n=79)$ en pareja, pero no convivia, el $15.8 \%(n=65)$ unido y conviviendo, sin estar casado legalmente, el 3.2\% $(n=13)$ casados legalmente y, por último, el $0.5 \%(n=2)$ divorciado legalmente.

\section{Instrumentos}

Cuestionario sociodemográfico. En el mismo se indagaron datos varios, tales como: edad, sexo, lugar de nacimiento, lugar de residencia, nivel socioeconómico, etc.

Big Five Inventory (BFI, John, 1990). Consiste en un instrumento de 44 ítems que evalúa los cinco grandes rasgos de personalidad (extraversión, agradabilidad, responsabilidad, neuroticismo, apertura a la experiencia). La prueba es un derivado de una prueba de adjetivos de personalidad, convertidos en frases cortas para facilitar la comprensión de los elementos de la prueba. El autor de la técnica demostró su validez y fiabilidad en grupos de población general adulta norteamericana. Esos estudios verificaron la validez concurrente con otros instrumentos reconocidos que evalúan personalidad. Los estudios locales verificaron la validez factorial de los instrumentos para población adolescente, población adulta no consultante y población militar (Castro Solano, 2005; Castro Solano \& Casullo, 2001). En todos los casos se obtuvo un modelo de cinco factores que explicaban alrededor del $50 \%$ de la variancia de las puntuaciones. En cuanto a la fiabilidad en población adulta argentina, se obtuvieron coeficientes en el rango de .66 a .77.

Mental Health Continuum - Short Form (MHC-SF, Keyes, 2006). Este instrumento de 14 items evalúa el grado de: a) Bienestar emocional (items 1, 2 y 3), entendido en términos de afectos positivos y satisfacción con la vida (bienestar hedónico); b) Bienestar social (incluye las facetas de aceptación, actualización, contribución social, coherencia e integración social) (items 4 a 8); c) Bienestar psicológico (items 9 a 14) en términos de la teoría de Ryff (autonomía, control, crecimiento personal, relaciones personales, autoaceptación y propósito). Estudios locales permitieron confirmar su fiabilidad y validez (Casullo \& Castro Solano, 2000).

Escala de Metas de Aprendizaje, Población Militar (EMA-M; Castro Solano $\&$ Casullo, 2002). Esta escala evalúa las prioridades que tienen los estudiantes al momento de abordar las materias del núcleo académico de grado, basada en las propuestas de Alonso Tapia (1997) para el estudio de la motivación académica. La misma consta de 11 elementos y permite evaluar tres tipos de orientaciones motivacionales (metas en relación con el aprendizaje y el dominio de los conocimientos, metas externas-aversivas y metas en relación con el lucimiento). El instrumento cuenta, asimismo, con características psicométricas adecuadas. Su fiabilidad para población militar Argentina se encuentra entre los valores .65 a .70 y permite predecir de forma bastante adecuada la elección vocacional en función de los intereses según áreas del conocimiento para este tipo de estudiantes.

\section{Procedimiento}

Se administró una batería de pruebas conformada por un cuestionario sociodemográfico, The Mental Health Continuum - Short Form, la Escala de Metas de Aprendizaje - Población Militar y un consentimiento informado a un grupo amplio de cadetes de la fuerza de seguridad de $1^{\text {er }}$ año en un aula acondicionada para tal efecto. Se entregaron las pruebas en un cuadernillo, a 
la vez que se dio una explicación verbal de las consignas instruidas. De igual manera, se realizó una breve charla introductoria a cargo del personal policial superior, destacando no solo la importancia del estudio sino también la veracidad de las respuestas. Además, se explicaron técnicamente los aspectos psicológicos implicados en dicha evaluación. La administración tuvo una duración promedio de 1 hora y 45 minutos. Los datos fueron cargados en el paquete estadístico SPSS 25.0.

\section{Análisis de datos}

En primer lugar, con objeto de explorar la relación entre las variables, se realizaron correlaciones de Pearson entre el bienestar y sus dimensiones con los rasgos de personalidad y con la escala de metas del aprendizaje; siguiendo los criterios de Cohen (1992), en donde se consideran y analizan con mayor profundidad los resultados que cumplan el tamaño del efecto moderado $(r \geq$ .30) a amplio $(r \geq .50)$. .

En segundo lugar, se realizó una regresión lineal múltiple para identificar si existía una influencia de los rasgos y metas de aprendizaje sobre el bienestar. Para esto se ubicó a los rasgos y las metas junto a sus factores como variables independientes y al bienestar como variables dependientes para el análisis de regresión.

Finalmente, se buscó analizar si existían diferencias significativas entre el bienestar y sus dimensiones y las variables sociodemográficas sexo y edad (Grupo 1: 19-22 años; Grupo 2: 23-25 años; Grupo 3: 26-30 años). Para ello se empleó Prueba $t$ para muestras independientes y ANOVA.

\section{RESULTADOS}

En primer lugar, se analizó si el bienestar se relaciona con la personalidad (tabla 1), para lo cual se llevó a cabo una correlación de Pearson, siguiendo los criterios de Cohen (1992), en donde se consideran y analizan los resultados que cumplan el tamaño del efecto moderado $(r \geq .30)$ a amplio $(r \geq$ .50). Se puede apreciar que existen correlaciones estadísticamente significativas entre el bienestar y sus dimensiones con los rasgos de personalidad. Tomando los criterios de Cohen (1992), se encontraron 14 correlaciones que presentan tamaño del efecto moderado.

Se halló que bienestar total correlaciona de manera negativa moderada $(r$ $\geq .30$ a .50$)$ con neuroticismo $(r=-.44 ; p<.001)$; de igual manera se encontró que dicho constructo correlaciona de manera positiva moderada con los rasgos: extraversión $(r=.36 ; p<.001)$; agradabilidad $(r=.40 ; p<.001) \mathrm{y}$ responsabilidad $(r=.45 ; p<.001)$. También correlaciona con apertura a la experiencia $(r=.20 ; p<.001)$. No obstante, su tamaño del efecto es pequeño $(r$ $\geq .10$ a.30).

En cuanto a las dimensiones del bienestar, se halló que el bienestar emocional correlaciona de manera positiva moderada $(r \geq .30$ a .50$)$ con los rasgos: extraversión $(r=.31 ; p<.001)$ y responsabilidad $(r=.33 ; \mathrm{p}<.001)$; también correlaciona de manera positiva con efecto pequeño con los rasgos: apertura a la experiencia $(r=.16 ; p<.001)$ y responsabilidad $(r=.28 ; p<.001)$. Finalmente correlaciona de manera negativa moderada $(r \geq .30$ a .50$)$ con neuroticismo $(r=-.39 ; p<.001)$.

Con respecto a la dimensión bienestar social se encontró que correlaciona de manera positiva moderada $(r \geq .30$ a .50) con los rasgos 
agradabilidad $(r=.37 ; p<.001)$ y responsabilidad $(r=.37 ; p<.001)$; también correlaciona de manera positiva con efecto pequeño $(r \geq .10$ a.30) con los rasgos: apertura a la experiencia $(r=.13 ; \mathrm{p}<.001)$ y extraversión $(r=.26 ; p<$ $.001)$. Finalmente correlaciona de manera negativa moderada ( $r \geq .30$ a .50) con neuroticismo $(r=-.32 ; p<.001)$.

Por último, la dimensión bienestar psicológico correlaciona de manera positiva moderada $(r \geq .30$ a .50) con los rasgos extraversión $(r=.38 ; p<.001)$; agradabilidad $(r=.37 ; p<.001)$ y responsabilidad $(r=.45 ; p=.001)$; también correlaciona de manera positiva con efecto pequeño $(r \geq .10$ a.30) con los rasgos: apertura a la experiencia $(r=.25 ; p<.001)$. Por último, correlaciona de manera negativa moderada con neuroticismo $(r=-.47 ; p<.001)$.

Tabla 1.

Relación entre bienestar y personalidad.

\begin{tabular}{lcccc}
\hline & $\begin{array}{c}\text { Bienestar } \\
\text { total }\end{array}$ & $\begin{array}{c}\text { Bienestar } \\
\text { emocional }\end{array}$ & $\begin{array}{c}\text { Bienestar } \\
\text { social }\end{array}$ & $\begin{array}{c}\text { Bienestar } \\
\text { psicológico }\end{array}$ \\
\hline Neuroticismo & $-.44^{* *}$ & $-.39^{* *}$ & $-.32^{* *}$ & $-.47^{* *}$ \\
Extraversión & $.36^{* *}$ & $.31^{* *}$ & $.26^{* *}$ & $.38^{* *}$ \\
Apertura a la experiencia & $.20^{* *}$ & $.16^{* *}$ & $.13^{* *}$ & $.25^{* *}$ \\
Agradabilidad & $.40^{* *}$ & $.28^{* *}$ & $.37^{* *}$ & $.37^{* *}$ \\
Responsabilidad & $.45^{* *}$ & $.33^{* *}$ & $.37^{* *}$ & $.45^{* *}$ \\
\hline
\end{tabular}

${ }^{\star *} p<.01$; En negrita tamaño del efecto moderado ( $\left.\geq .30\right)$ a amplio $(\geq .50)$.

En segundo lugar, se analizó si el bienestar se relaciona con las metas de aprendizaje (tabla 2) donde, al igual que en el estudio anterior, se llevó a cabo una correlación de Pearson siguiendo los criterios de Cohen (1992) mencionados anteriormente. Se puede apreciar que existen correlaciones estadísticamente significativas entre el bienestar y sus dimensiones con las dimensione de metas de aprendizaje y el dominio de los conocimientos. No obstante, no se encontraron correlaciones que presentan tamaño del efecto moderado, observándose que todas presentan un tamaño del efecto pequeño.

Se halló que bienestar total correlaciona de manera negativa con efecto pequeño $(r \geq .10$ a. 30) con metas externas-aversivas $(r=-.15 ; p=.003) \mathrm{y}$ correlaciona de manera positiva con efecto pequeño $(r \geq .10$ a .30$)$ con metas de aprendizaje y el dominio de los conocimientos $(r=.24 ; p<.001)$. En cuanto a las dimensiones del bienestar, se halló que el bienestar emocional correlaciona de manera positiva con efecto pequeño $(r \geq .10$ a .30), con la dimensión metas en relación con el aprendizaje y el dominio de los conocimientos $(r=.20 ; p<.001)$, y correlaciona de manera negativa con efecto pequeño $(r \geq .10$ a .30) y con las dimensiones metas externas-aversivas $(r=-$ $.21 ; p<.001)$ y metas en relación con el lucimiento $(r=-.12 ; p=.010)$.

Con respecto a la dimensión bienestar social, se encontró una correlación positiva con efecto pequeño $(r \geq .10$ a .30) con metas de aprendizaje y el dominio de los conocimientos $(r=.16 ; p<.001)$ y una correlación negativa con efecto pequeño $(r \geq .10$ a.30) con metas externasaversivas $(r=-.12 ; p=.015)$. Por último, en cuanto a la dimensión bienestar psicológico, se observó una correlación positiva con efecto pequeño $(r \geq .10$ a .30) con metas en relación con el aprendizaje y el dominio de los conocimientos $(r=.20 ; p<.001)$ y una correlación negativa con efecto pequeño $(r \geq .10$ a .30$)$ con metas externas-aversivas $(r=-.10 ; p=.040)$. 
Tabla 2.

Relación entre bienestar y metas de aprendizaje.

\begin{tabular}{lcccc}
\hline & $\begin{array}{c}\text { Bienestar } \\
\text { total }\end{array}$ & $\begin{array}{c}\text { Bienestar } \\
\text { emocional }\end{array}$ & $\begin{array}{c}\text { Bienestar } \\
\text { social }\end{array}$ & $\begin{array}{c}\text { Bienestar } \\
\text { psicológico }\end{array}$ \\
\hline Metas en relación con el aprendizaje y & $.21^{* *}$ & $.20^{* *}$ & $.16^{* *}$ & $.20^{* *}$ \\
el dominio de los conocimientos & $-.15^{* *}$ & $-.21^{* *}$ & $-.12^{*}$ & $-.10^{*}$ \\
Metas externas-aversivas & -.08 & $-.12^{* *}$ & -.03 & -.09 \\
\hline Metas en relación con el lucimiento & & & &
\end{tabular}

En tercer lugar, para investigar si las variables Metas de Aprendizaje (aprendizaje y dominio de los conocimientos; metas aversivas externas y metas de lucimiento) y personalidad (extraversión, agradabilidad, responsabilidad, neuroticismo y apertura a la experiencia) predicen el bienestar de los cadetes aspirantes a policías, se realizó una regresión lineal múltiple, donde la prueba de Durbin-Watson indicó independencia de errores (1.806). Con respecto a la prueba de hipótesis para dicho modelo, se observó que las variables independientes rasgos de personalidad y metas de aprendizaje predicen el $31 \%$ de la variable dependiente bienestar $\left(r^{2}=.3 .18\right)$. Asimismo, se observó que los mayores predictores positivos del bienestar fueron extraversión $(t=3.412 ; \beta=$ $.157 ; p<.001)$; agradabilidad $(t=2.901 ; \beta=.144 ; p=.005)$, responsabilidad $(t$ $=3.851 ; \beta=.195 ; p<.001) \mathrm{y}$ metas de aprendizaje $\mathrm{y}$ dominio de los conocimientos $(t=2.446 ; \beta=.103 ; p=.003)$. De igual manera, se observó que el único predictor negativo fue el rasgo de personalidad neuroticismo $(t=-$ $3.555 ; \beta=-.184 ; p<.001)$.

Tabla 3.

Predictores de Bienestar.

\begin{tabular}{lcccc}
\hline Predictores & $\boldsymbol{B}$ & $\boldsymbol{E E}(\boldsymbol{B})$ & $\boldsymbol{\beta}$ & $\boldsymbol{t}$ \\
\hline Neuroticismo & -0.425 & 0.120 & -0.184 & $-3.555^{* *}$ \\
Extraversión & 0.340 & 0.100 & 0.157 & $3.412^{* *}$ \\
Apertura a la experiencia & 0.152 & 0.094 & 0.069 & $1.605^{\text {ns }}$ \\
Agradabilidad & 0.352 & 0.121 & 0.144 & $2.901^{\text {** }}$ \\
Responsabilidad & 0.397 & 0.103 & 0.195 & $3.851^{\text {** }}$ \\
Metas en relación con el aprendizaje y el & 0.448 & 0.183 & 0.103 & $2.446^{*}$ \\
dominio de los conocimientos & -0.278 & 0.197 & -0.064 & $-1.410^{\text {ns }}$ \\
Metas externas-aversivas & -0.013 & 0.206 & -0.003 & $-0.061^{\text {ns }}$ \\
\hline Metas en relación con el lucimiento & & & &
\end{tabular}

${ }^{* *} p<.01 ;{ }^{*} p<.05 ; n s=$ no significativo

En cuarto lugar, para analizar si existen diferencias en el bienestar y sus dimensiones en función del sexo, se realizó una prueba $t$ para muestras independientes (tabla 4), donde se observó una diferencia estadísticamente significativa en la dimensión bienestar social $\left(t_{(410)}=1.816 ; p=.041\right)$, donde los hombres $(M=15.95)$ puntuaron significativamente más alto que las mujeres $(M=14.92)$. 
Tabla 4.

Diferencias en el bienestar según género.

\begin{tabular}{lccc}
\hline & \multicolumn{2}{c}{ Género } & $t_{(410)}$ \\
\cline { 2 - 4 } & Masculino & Femenino & \\
& $M(D E)$ & $M(D E)$ & \\
\hline Bienestar psicológico & $24.69(4.86)$ & $24.87(4.20)$ & $-0.396^{n s}$ \\
Bienestar emocional & $12.93(1.97)$ & $12.80(1.93)$ & $0.691^{n s}$ \\
Bienestar social & $15.95(5.71)$ & $14.92(5.58)$ & $1.816^{*}$ \\
Bienestar total & $53.57(11.15)$ & $52.59(10.15)$ & $0.912^{n s}$ \\
\hline${ }^{*} p<0.05 ; n s:$ no significativo & & &
\end{tabular}

Finalmente, para analizar si existen diferencias en el bienestar y sus dimensiones según edad, se realizó la prueba de ANOVA con prueba Post Hoc de Bonferroni, para lo cual se dividió la variable edad en tres grupos (Grupo 1: 19-22 años; Grupo 2: 23-25 años; Grupo 3: 26-30 años). Con respecto a la dimensión bienestar psicológico, se encontraron diferencias significativas según edad $\left(F_{(2,411)}=3,385 ; p=.034\right) \mathrm{y}$, de igual manera, en las comparaciones post hoc se observaron diferencias significativas en los 3 grupos de edad: grupo $1(M=23.98)$, grupo $2(M=25.24)$ y grupo $3(M=25.27)$, lo que significa que el grupo de mayor edad presenta mayores niveles de bienestar psicológico. En cuanto a bienestar total $\left(F_{(2,411)}=3,608 ; p=.028\right)$, las comparaciones arrojaron diferencias significativas entre el grupo $1(M=51.28)$ y el grupo $2(M$ $=54.28$ ), evidenciando que, a mayor edad, mayor es el bienestar total.

Tabla 5.

Diferencias en bienestar según edad.

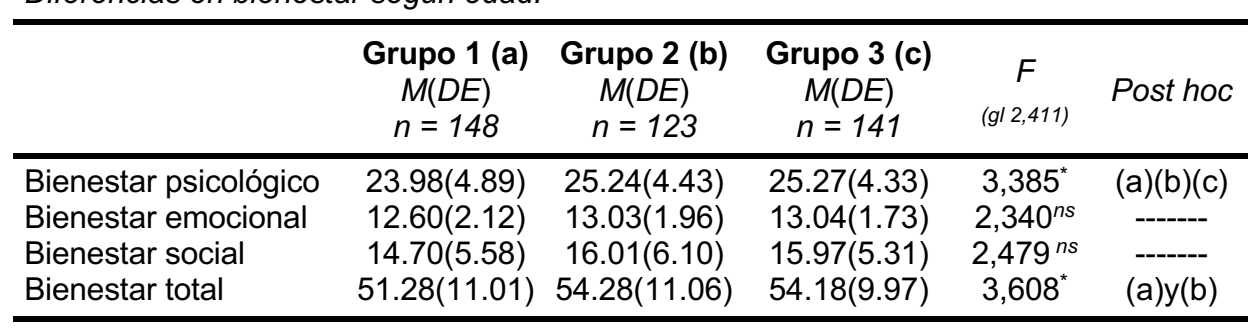

${ }^{*} p<.05 ; n s:$ no significativo

\section{COMENTARIOS}

Los estudios citados en esta investigación sostienen que el bienestar es una de las variables que más influye en el rendimiento académico y en el desarrollo efectivo en el ámbito académico/laboral y, a la vez, resaltan que el tipo de bienestar tiene relación con variables personológicas y motivacionales. Por ello, esta investigación buscó analizar si las variables metas de aprendizaje (metas de aprendizaje y dominio de los conocimientos; metas aversivas externas y metas de lucimiento) y personalidad (extraversión, agradabilidad, responsabilidad, neuroticismo y apertura a la experiencia) predicen el bienestar en cadetes de policías. Asimismo, se buscó evaluar si existían diferencias significativas entre el bienestar y las variables sociodemográficas sexo y edad en cadetes de policía.

En primer lugar, se analizó bienestar y personalidad, sobre lo cual se observó que el bienestar total correlaciona de manera negativa con el rasgo neuroticismo y de manera positiva con los rasgos extraversión, agradabilidad, responsabilidad y apertura a la experiencia. 
En cuanto a las dimensiones del bienestar, se halló que el bienestar psicológico correlaciona de manera positiva con extraversión, responsabilidad y apertura a la experiencia y de manera negativa con neuroticismo. Con respecto a la dimensión bienestar social, se encontró que esta correlaciona de manera positiva con los rasgos agradabilidad, responsabilidad, apertura a la experiencia y extraversión. Lo contrario sucedió con la dimensión neuroticismo, con la cual correlaciona de manera negativa. Por último, la dimensión bienestar emocional correlaciona de manera positiva con los rasgos extraversión, agradabilidad, responsabilidad y apertura a la experiencia y de manera negativa con neuroticismo. En este sentido, los resultados concuerdan con lo reportado por Schmutte y Ryff (1997), quienes, de manera similar, observaron que los rasgos de personalidad correlacionan de manera positiva con el bienestar, a excepción del rasgo neuroticismo, que lo hace de manera negativa. Esto significa que aquellos individuos con alta responsabilidad presentarian mayor autonomía y dominio del entorno, lo que daria lugar a una mejor autoaceptación. Por otra parte, los altos niveles de agradabilidad se relacionan con las relaciones positivas, donde los individuos establecen vínculos satisfactorios basados en el apoyo y la calidez. A su vez, las autoras mencionan que la extraversión es otro facilitador de las relaciones positivas.

Dentro de este marco, Garbarino, Chiorri y Magnavita (2014) sostienen que los rasgos de la personalidad normal podrian influir sobre el rendimiento de los futuros líderes militares y cadetes a policia, puesto que, hasta el momento, las investigaciones se habian centrado en la consideración de los rasgos patológicos de la personalidad. Por ejemplo, dentro del modelo de personalidad Big Five, el rasgo responsabilidad fue uno de los factores predictor del éxito académico en el rendimiento de los estudiantes de secundaria y universitarios (Noftle \& Robins, 2007). En relación con esto, Castro Solano y Fernández Liporace (2005) hallaron que bajos niveles de responsabilidad y altos niveles de extraversión se relacionan con niveles bajos de rendimiento académico y militar durante el primer año de entrenamiento. Depaula y Azzollini (2013) sostienen que las personalidades responsables, con fuerte voluntad e inteligencia cultural reflexiva-motivacional, constituyen predictores del óptimo rendimiento.

En segundo lugar, se buscó analizar si existe relación entre el bienestar y sus dimensiones con las metas de aprendizaje. Se halló que bienestar total correlaciona de manera negativa con metas externas-aversivas y correlaciona de manera positiva con metas en relación con el aprendizaje y el dominio de los conocimientos. En cuanto a las dimensiones del bienestar, se encontró que el bienestar psicológico correlaciona de manera positiva con la dimensión metas en relación con el aprendizaje y el dominio de los conocimientos, y correlaciona de manera negativa con las dimensiones metas externasaversivas y metas en relación con el lucimiento.

Con respecto a la dimensión bienestar social, se encontró que esta correlaciona de manera positiva con metas en relación con el aprendizaje y el dominio de los conocimientos, y de manera negativa con metas externasaversivas. Por último, respecto a la dimensión bienestar emocional se observó que correlaciona de manera positiva con metas en relación con el aprendizaje y el dominio de los conocimientos, y correlaciona de manera negativa con metas externas-aversivas.

De esta manera, las metas en relación con el aprendizaje y el dominio de los conocimientos tienen que ver con un aprendizaje basado en el deseo de 
aprender en sí mismo, es decir, el valor reside en los conocimientos a adquirir, por la satisfacción personal que esto produce o por el deseo de dominar algún aspecto del conocimiento, sin buscar algo a cambio (Castro Solano, 2005). En oposición, las metas aversivas externas refieren al valor que se les otorga a las consecuencias resultantes del estudio y no al aprendizaje en sí mismo, donde justamente se manifiesta aversión por el objeto de conocimiento. Por otra parte, las metas de lucimiento refieren al aprendizaje que tiene base en la búsqueda de aceptación y reconocimiento de otros, ya sea por parte de profesores, ya sea por parte de instructores. En este sentido, este último tipo de metas se caracterizan por buscar la valoración de un superior, por ejemplo (Depaula, 2012).

En relación a lo expuesto, Depaula (2012) destaca la relevancia de estudiar la motivación por el aprendizaje en estudiantes del ámbito de las fuerzas de seguridad, ya que en sus estudios ha hallado que las metas aversivas externas predicen negativamente el rendimiento general, demostrando que los estudiantes que reciben formación académica y están motivados extrínsecamente, sienten que estudiar es una obligación impuesta de la cual deben librarse en el menor tiempo posible y/o para lograr una permanencia en la institución.

Con respecto a detectar si las variables metas de aprendizaje (metas de aprendizaje y el dominio de los conocimientos; metas aversivas externas y metas de lucimiento) y personalidad (extraversión, agradabilidad, responsabilidad, neuroticismo y apertura a la experiencia) son predictoras del bienestar, se aplicó un análisis de regresión lineal múltiple para distinguir los mayores predictores, sobre lo que se observó que los mayores predictores positivos del bienestar fueron extraversión, agradabilidad, responsabilidad (personalidad) y metas relacionadas al aprendizaje y el dominio de los conocimientos (metas de aprendizaje). De igual manera, se encontró que el único predictor negativo fue el rasgo de personalidad neuroticismo (personalidad). Por último, las dimensiones que no son predictoras del bienestar fueron: apertura a la experiencia (personalidad), metas externasaversivas y metas en relación con el lucimiento (metas de aprendizaje). Estos resultados son congruentes con los reportados en investigaciones anteriores, en las cuales se observó que la responsabilidad, el neuroticismo y la extraversión son los mayores predictores de las dimensiones del bienestar (Bardi \& Ryff, 2007; Grant et al., 2009; Keyes et al., 2002; Schmutte \& Ryff, 1997).

Con relación a si existen diferencias entre el bienestar y sus dimensiones según sexo, se observó una diferencia estadísticamente significativa en la dimensión bienestar social, donde los hombres puntuaron más alto que las mujeres. Si bien existe en la literatura una tendencia a observar diferencias en el bienestar según sexo (en las cuales las mujeres tienden a puntuar más alto que los hombres), las mismas varian según los autores (García Alandete, 2013; Mayordomo et. al, 2016; Muratori et al., 2015; Zubieta et. al, 2012;).

Finalmente, con relación al último estudio de esta investigación, en el cual se propuso analizar si existen diferencias en el bienestar y sus dimensiones según edad, se halló que en la dimensión bienestar psicológico existen diferencias significativas según edad entre los tres grupos, donde el grupo de mayor edad presenta mayores niveles de bienestar psicológico. En cuanto al bienestar total, las comparaciones arrojaron diferencias significativas entre el grupo de menor edad, respecto al segundo grupo, lo que 
significa que, a mayor edad mayor, es el bienestar total. Estos resultados son congruentes con investigaciones anteriores donde, de manera similar, se observó que este varía en función de la edad, aclarando que, a mayor edad, mayor bienestar (Mayordomo et al., 2016; Muratori et al., 2015; Zubieta et al., 2012;). Como bien afirma Keyes (2002), esto puede deberse al desarrollo de las dimensiones del bienestar conforme al paso de los años, donde el propósito en la vida, el crecimiento personal y el desarrollo del potencial humano acrecientan a lo largo de la vida.

En relación con los resultados, Castro Solano (2005) destaca la importancia de evaluar las características que poseen los cadetes para poder predecir el éxito educativo, debido a que conocer las variables implicadas es útil para la selección de candidatos idóneos para ocupar posiciones en las fuerzas armadas.

Asimismo, el estudio y análisis de los factores que predicen el rendimiento en instituciones policiales es una temática de gran relevancia, ya que la evaluación psicológica de los estudiantes permitirá no solo reelaborar los programas educativos, sino también identificar jóvenes en riesgo de fracaso a causa de factores psicopatológicos, predecir el rendimiento exitoso, evaluar el costo económico y el impacto social de la carrera y seleccionar adecuadamente los recursos humanos (Bailey, 2013).

Sin embargo, hasta el momento la psicología argentina no ha estudiado los factores comentados a fin de contribuir al mejor entrenamiento, capacitación y selección de buenos policias. Cabe destacar que se han encontrado pocas investigaciones empíricas en el área de la formación de cadetes a policía (Bailey, 2013; Klafehn et al., 2014), ya que el foco de atención de esta se centró en la identificación de características desadaptativas de personalidad, en la presencia de trastornos psicológicos y en los aspectos adaptativos del buen desempeño en militares.

No obstante, vale aclarar que estos resultados deben ser interpretados a la luz de algunas limitaciones importantes. Primero, el diseño transversal con el cual se llevó a cabo la presente investigación no es capaz abordar directamente cuestiones de direccionalidad o cambio de desarrollo en el tiempo. Asimismo, las escasas investigaciones que se presentan en la población seleccionada dificultan el relevo de antecedentes para llevar a cabo una comparación de los resultados obtenidos. Sin embargo, vale destacar que el presente estudio representa un primer intento de establecer una relación entre bienestar con metas de aprendizaje y personalidad en cadetes de las fuerzas de seguridad en Argentina, Buenos Aires.

En segundo lugar, la variación de estos resultados pudo deberse al tipo de muestra, ya que la misma abarcó un rango amplio de edad, se administró a una muestra especifica de cadetes de policías y solo se administró a una institución pública, donde la mayoría de los encuestados pertenecian a la Provincia de Buenos Aires, Argentina. No obstante, cabe aclarar que es una muestra no probabilística de sujetos voluntarios.

En tercer lugar, el desconocimiento de las evaluaciones previas realizadas para la selección de cadetes al momento de ingresar a la institución genera la imposibilidad de conocer o armar un perfil psicológico acerca de las cualidades que debe tener en un aspirante a cadete de policía. De igual manera, otra de las dificultades que presentó esta investigación fue la desigualdad de los grupos con respecto al género. $Y$, por último, una de las 
limitaciones más relevantes del presente estudio fue no utilizar la escala de metas de aprendizaje adaptada a dicha población.

Futuras líneas de investigación deberán abordar dicha temática con otras variables de tipo cognitivas, sociales, emocionales, entre otras, así como hacer estudios en diversas fuerzas de seguridad, ya que los pocos estudios hallados están realizados en población militar. Dentro de esta misma línea, resultaria interesante hacer un estudio en donde se comparen estas variables con el rendimiento académico. De igual manera, sería interesante realizar estudios de tipo prepos prueba, donde se mida el rendimiento durante la formación y luego durante la especialización en distintos ámbitos de inserción que ofrecen las fuerzas de seguridad en nuestro país. También, se sugiere realizar otros tipos de análisis estadísticos más complejos como ecuaciones lineales, modelo de ecuaciones, entre otros.

Finalmente, vale aclarar que, aunque los resultados obtenidos en esta investigación no son concluyentes pero sí significativos, estos pueden servir para diversos educadores y/o profesionales para reelaborar el proceso de elección de los aspirantes a cadetes a policías.

\section{REFERENCIAS}

Adler, A. B. (2013). Resilience in a military occupational health context: directions for future research. En R. R. Sinclair \& T. W. Britt (Eds.), Building psychological resilience in military personnel: theory and practice (pp. 223 - 235). Washington: American Psychological Association. https://doi.org/10.1037/14190-010

Alonso Tapia, J. (1997). Orientación Educativa. Teoría, evaluación e intervención. Madrid: Sintesis.

Ames, C. (1992). Classrooms: Goals, structures, and student motivation. Journal of Educational Psychology, 84(3), 261-271. https://doi.org/10.1037/0022-0663.84.3.261

Bailey, M. S. (2013). Tropical skin diseases in British military personnel. Journal of the Royal Army Medical Corps, 159(3), 224-228. https://doi.org/10.1136/jramc-2013-000124

Bardera, M. P., Garcia-Silgo, M. \& Pastor, A. (2014). Stress management in the armed forces. Journal of the Spanish Institute for Strategic Studies, 4, 1-24.

Bardi, A. \& Ryff, C. D. (2007). Interactive effects of traits on adjustment to a life transition. Journal of Personality, 75(5), 955-983. https://doi.org/10.1111/j.1467-6494.2007.00462.x

Benatuil, D., \& Solano, A. C. (2007). La inteligencia práctica como predictor del rendimiento de cadetes militares. Anuario de psicología, 38(2), 305-320.

Burris, J. L., Brechting, E. H., Salsman, J., \& Carlson, C. R. (2009). Factors associated with the psychological well-being and distress of university students. Journal of American College Health, 57(5), 536-544. https://doi.org/10.3200/JACH.57.5.536-544

Castro Solano, A. \& Casullo, M. M. (2001). Rasgos de personalidad, bienestar psicológico y rendimiento académico en adolescentes argentinos. Interdisciplinaria Revista de Psicología y Ciencias Afines, 18, 65-85.

Castro Solano, A. \& Fernández Liporace, M. (2005). Predictores para la selección de cadetes en instituciones militares. Psykhe, 14(1), 17-30. https://doi.org/10.4067/S0718-22282005000100002

Castro Solano, A. (2005). Técnicas de evaluación psicológica en los ámbitos militares. Buenos Aires: Paidós.

Casullo, M. M. \& Castro Solano, A. (2000). Evaluación del bienestar psicológico en estudiantes adolescentes argentinos. Revista de Psicología de la Pontificia Universidad Católica de Perú, 18(1), 36-68.

Cohen, J. (1992). A power primer. Psychological bulletin, 112(1), 155-159. https://doi.org/10.1037/00332909.112.1.155

Compton, W. C. (1998). Measures of mental health and a five-factor theory of personality. Psychological Reports, 83(1), 371-381. https://doi.org/10.2466/pr0.1998.83.1.371

Cooke, R., Bewick, B., Barkham, M., Bradley, M., \& Audin, K. (2006). Measuring, monitoring and managing the psychological well-being of first year university students. British Journal of Guidance and Counselling, 34(4), 505-517. https://doi.org/10.1080/03069880600942624

Costa, P. T. \& McCrae, R. R. (1992). Revised NEO Personality Inventory and NEO Five- Factor Inventory: Professional manual. Odessa, FL: Psychological Assessment Resources.

Deci, E. L. \& Ryan, R. M. (2000). The "what" and "why" of goal pursuits: Human needs and the selfdetermination of behavior. Psychological Inquiry, 11, 319-338. https://doi.org/10.1207/S15327965PLI1104_01

Depaula, P. D., (2012). Predictores globales de la performance de estudiantes militares. Revista Ciencias Psicológicas, 6, 135-148.

Depaula, P. D. \& Azzollini, S. C. (2013). Análisis del modelo Big Five de la personalidad como predictor de la inteligencia cultural. PSIENCIA: Revista Latinoamericana de Ciencia Psicológica, 5(1), 35-43. https://doi.org/10.5872/psiencia/5.1.24

Diener, E. (1984). Subjective well-being. Psychological Bulletin, 95(3), 542-575. 
http://dx.doi.org/10.1037/0033-2909.95.3.542

Garbarino, S., Chiorri, C., \& Magnavita, N. (2014). Personality traits of the five-factor model are associated with work-related stress in special force police officers. International archives of occupational and environmental health, 87(3), 295-306. https://doi.org/10.1007/s00420-013-0861-1

García Alandete, J. (2013). Bienestar psicológico, edad y género en universitarios españoles. Salud \& Sociedad, 4(1), 48-58. https://doi.org/10.22199/S07187475.2013.0001.00004

Grant, S., Langan-Fox, J., \& Anglim, J. (2009). The big five traits as predictor of subjective and psychological well-being. Psychological Reports, 105(1), 205-231. https://doi.org/ 10.2466/PR0.105.1.205-231

John, O. (1990). The five-factor taxonomy: Dimension of personality in the natural language and in questionnaires. In: L. Pervin (Ed.), Handbook of personality: Theory and Research. New York: Guilford Press.

John, O. P., Donahue, E. M., \& Kentle, R. L. (1991). The Big Five Inventory - Versions 4a and 54. Berkeley: University of California, Berkeley, Institute of Personality and Social Research. https://doi.org/10.1037/t07550-000

Kasser, T. \& Ryan, R. M. (1996). Further examining the American dream: Differential correlates of intrinsic and extrinsic goals. Personality and Social Psychology Bulletin, 22, 80-87. https://doi.org/10.1177/0146167296223006

Keyes, C. L. M. (1998). Social well-being. Social Psychology Quarterly, 61, 121-140. https://doi.org/10.2307/2787065

Keyes, C. L. (2002). The mental health continuum: From languishing to flourishing in life. Journal of health and social behavior, 43(2), 207-222. https://doi.org/10.2307/3090197

Keyes, C. L. M. (2006). Mental health in adolescence: is America's youth flourishing? American Journal of Orthopsychiatry, 76(3), 395. https://doi.org/10.1037/0002-9432.76.3.395

Keyes, C. L. M., Shmotkin, D., \& Ryff, C. D. (2002). Optimizing well-being: the empirical encounter of two traditions. Journal of Personality and Social Psychology, 82(6), 1007-1022. https://doi.org/10.1037/0022-3514.82.6.1007

Klafehn, J. L., Cai, D. A., Connelly, S., Mathieu, J. E., Maurer, T. J., Noe, R. A., \& Salazar, M. (2014). Soldier development following negative cross-cultural experiences: An integrated review of the literature. Estados Unidos: Army research institute for the behavioral and social sciences. https://doi.org/10.21236/ADA599261

Lepper, M. R. (1988). Motivational considerations in the study of instruction. Cognition and Instruction, 5(4), 289-309. https://doi.org/10.1207/s1532690xci0504_3

Mayordomo, T., Sales, A., Satorres, E. \& Meléndez, J. C. (2016). Bienestar psicológico en función de la etapa de vida, el sexo $\mathrm{y}$ su interacción. Pensamiento Psicológico, 14(2), 101-112. https://doi.org/10.11144/Javerianacali.PPSI14-2.bpfe

Muratori, M., Zubieta, E., Bobowik, M., Ubillos, S. \& González, J. L. (2015). Felicidad y Bienestar Psicológico: Estudio Comparativo Entre Argentina y España. Psykhe, 24(2) 1-18. https://doi.org/10.7764/psykhe.24.2.900

Noftle, E. E., \& Robins, R. W. (2007). Personality predictors of academic outcomes: Big five correlates of GPA and SAT scores. Journal of Personality and Social Psychology, 93, 116-130. https://doi.org/10.1037/0022-3514.93.1.116

Ryan, R. M. \& Deci, E. L. (2000). Self-determination theory and the facilitation of intrinsic motivation, social development, and well-being. American Psychologist, 55, 68-78. https://doi.org/10.1037/0003066X.55.1.68

Ryan, R. M. \& Deci, E. L. (2001). On happiness and human potentials: a review of research on hedonic and eudaimonic well-being. Annual Review of Psychology, 52, 141-166. https://doi.org/10.1146/annurev.psych.52.1.141

Ryff, C. D. (1989). Happiness is everything or is it? explorations on the meaning of psychological well-being. Journal of personality and social psychology, 57(6), 1069-1081. https://doi.org/10.1037/00223514.57.6.1069

Schmutte, P. S. \& Ryff, C. D. (1997). Personality and well-being: reexamining methods and meanings. Journal of Personality and Social Psychology, 73(3), 549-559. https://doi.org/10.1037/00223514.73.3.549

Seligman, M. \& Csikszentmihalyi, M. (2000). Positive Psychology: An Introduction. American Psychologist, $55(1), 5-14$

Siegrist, J. (2003). Subjective well-being: new conceptual and methodological developments in health-related social sciences. ESF SCSS Exploratory Workshop on Income, Interactions and Subjective Well-being. Paris.

Wang, Z., Inslicht, S. S., Metzler, Y. T. J., Henn-Haase, C., McCaslin, S. E., Tong, H. \& Marmar, C. R. (2010). A prospective study of predictors of depression symptoms in police. Psychiatry Research, 175(3), 211-216. https://doi.org/10.1016/j.psychres.2008.11.010

Zubieta, E., Muratori, M., \& Fernández, O. (2012). Bienestar Subjetivo y Psicosocial: Explorando Diferencias De Género. Salud \& Sociedad, 3(1), 66-76. https://doi.org/10.22199/S07187475.2012.0001.00005

Recibido 07-12-2018 | Aceptado 27-03-2019

Este trabajo se encuentra bajo una Licencia Creative Commons Atribución 4.0 Internacional que permite a terceros utilizar lo publicado siempre que se dé el crédito pertinente a los autores y a Psicodebate 